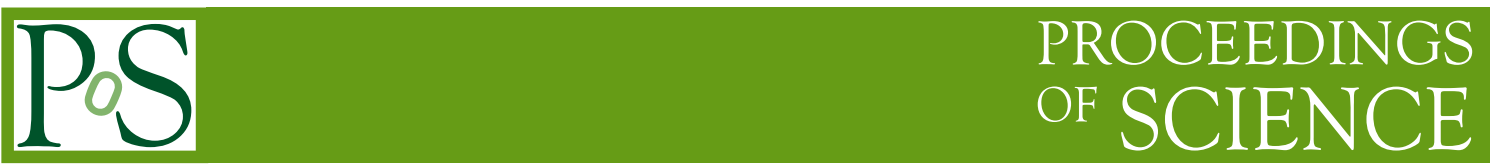

\title{
High-Energy Atmospheric Muons in IceCube and IceTop
}

\author{
The IceCube Collaboration ${ }^{\dagger}$ \\ ${ }^{\dagger}$ http://icecube.wisc.edu/collaboration/authors/icrc17_icecube \\ E-mail: frederik.tenholt@icecube.wisc.edu
}

\begin{abstract}
High-energy atmospheric muons can yield information about the prompt contribution to atmospheric lepton fluxes. Relevant to studying the flux of astrophysical neutrinos, this also complements results from collider experiments in the forward region. A machine-learning based selection has been developed, identifying high-energy $\left(E_{\mu} \gtrsim 1 \mathrm{TeV}\right)$ leading muons which dominate the energy losses detected in IceCube. The sample is then analyzed in two ways. First, the correlation between the muon energy in ice and the muon energy at its production in the atmosphere, which can be derived from simulations based on Monte-Carlo methods, is used for estimating the differential energy spectrum of atmospheric muons in the energy range between 6 and $400 \mathrm{TeV}$. The best-fit power law index describing the atmospheric muon flux is found to be consistent with the result of a previous analysis. Second, dedicated simulations are used to show a proof-of-concept for reconstructing the effective Feynman- $x$ of atmospheric muons by combining information from IceCube and IceTop. A robust correlation between true and reconstructed effective Feynman- $x$ is found, enabling future studies of this quantity with the IceCube Neutrino Observatory.
\end{abstract}

Corresponding authors: Frederik Tenholt ${ }^{* 1}$

${ }^{1}$ Fakultät für Physik \& Astronomie, Ruhr-Universität Bochum, D-44780 Bochum, Germany

35th International Cosmic Ray Conference - ICRC2017

10-20 July, 2017

Bexco, Busan, Korea

${ }^{*}$ Speaker. 


\section{Introduction}

IceCube is a $1 \mathrm{~km}^{3}$ neutrino detector installed in the ice at the geographic South Pole [1] between depths of $1450 \mathrm{~m}$ and $2450 \mathrm{~m}$, completed in 2010. Reconstruction of the direction, energy and flavor of the neutrinos relies on the optical detection of Cherenkov radiation emitted by charged particles produced in the interactions of neutrinos in the surrounding ice or the nearby bedrock. In 2013, the IceCube Collaboration found evidence for an astrophysical flux of neutrinos [2]. Since atmospheric leptons are the main background to such a flux, a detailed understanding of them is essential. One important aspect is the contribution of prompt decays from short-lived hadrons, that is, from charmed mesons and unflavored vector mesons [3]. Because of their short lifetime, these intermediate air shower particles usually decay before interacting, yielding harder lepton spectra than those induced by light mesons like $\pi^{ \pm}$and $\mathrm{K}^{ \pm}$[4]. With a rate of about $2100 \mathrm{~s}^{-1}$, muons originating from extended air showers are the most frequent particles triggering IceCube and hence represent the main background for the detection of neutrinos. At the same time, however, muons provide an excellent basis for measurements themselves. After a description of the simulations in Section 2 and a presentation of the machine-learning based selection used for selecting high-energy leading muons in Section 3, a measurement of the differential energy spectrum of high-energy atmospheric muons detected in IceCube and IceTop is presented and discussed in Section 4. The production of high-energy atmospheric muons depends on the energy $E_{\mathrm{cr}}$ of the primary cosmic ray inducing the extended air shower and the fraction of this energy that is transferred to the muon. We define this fraction as the effective Feynman- $x$ in the laboratory system:

$$
x_{\mathrm{F}, \mathrm{eff}}^{\mathrm{lab}} \equiv \frac{E_{\mu}}{E_{\mathrm{cr}}}
$$

with the muon energy $E_{\mu}$ at its production in the atmosphere. A machine-learning approach for reconstructing $x_{\mathrm{F}, \text { eff }}^{\mathrm{lab}}$ combining information from IceCube and IceTop is presented in Section 5, followed by the conclusions and an outlook in Section 6 .

\section{Simulations}

The starting point for all simulations used in this analysis are primary cosmic rays on the level of the atmosphere that are weighted to a model of the spectrum and composition of cosmic rays based on the available cosmic ray data. Here, the parametrization implementing the Hillas model with three populations and a mixed third population (H3a) [5] is used. The air shower development is then simulated using the CORSIKA [6] package with Sibyll 2.1 [7] for the high-energy and FLUKA $[8,9]$ for the low-energy hadronic interactions. The following simulation steps, including the propagation of the particles through the ice, the photon emission and the IceTop and IceCube detector simulations, are accomplished using standard IceCube software packages. Two different simulation types are used: standard IceCube simulations covering the five most important element groups $\mathrm{H}, \mathrm{He}, \mathrm{CNO}, \mathrm{MgAlSi}$ and Fe over an energy range of $5<\log _{10} E_{\mathrm{cr}} / \mathrm{GeV}<11$ and with about $3.5 \times 10^{9}$ simulated showers. These simulations are used for the event selection described in Section 3 and the unfolding of the muon energy spectrum in Section 4. The second type of simulations uses a kill-threshold principle, where only showers that are capable of producing a 
high-energy muon exceeding a certain energy and/or effective Feynman- $x$ are propagated, leading to a better efficiency in simulating showers with a single high-energy muon. Also, on the contrary to the standard simulations, the kill-threshold simulations contain the electromagnetic component of the shower as well as the IceTop response, which are necessary for the machine-learning algorithm presented in Section 5.2.

\section{Event Selection and Data Sample}

In this section, the event selection used in Section 4 and 5 is presented. After cuts that ensure a minimum quality of the sample (Section 3.1), a machine-learning approach implementing a random forest [10] classification is described in Section 3.2. The data sample used in Section 4 corresponds to a detector livetime of 168.2 days, which is also chosen as the normalization of the simulations.

\subsection{Minimum Quality Cuts}

In order to obtain the desired sample containing high-energy events with successful reconstructions for direction and energy that traverse IceCube as well as IceTop, the minimum quality cuts in Table 1 are applied to data and simulations.

\begin{tabular}{lll}
\hline No. & Cut & Purpose \\
\hline (1) & $Q_{\text {tot }}>1000$ photo-electrons & Select high-energy events \\
(2) & Directional reconstruction [11] successful & Required for (3) \\
(3) & $r_{\text {IceTop }}<500 \mathrm{~m}$ & Select events traversing IceTop \\
(4) & Energy reconstruction [12] successful & Muon energy proxy \\
(5) & $L_{\text {track }} \geq 720 \mathrm{~m}$ & Remove short tracks \\
\hline
\end{tabular}

Table 1: Overview of the minimum quality cuts using the total charge $Q_{\text {tot }}$ deposited in IceCube, the distance $r_{\text {IceTop }}$ between the intersection of the trajectory with the surface plane and the center of IceTop and the track length $L_{\text {track }}$ inside the detector (as determined by the energy reconstruction [12]).

\subsection{Random Forest Classification}

High-energy track events in IceCube originating from atmospheric muons usually contain a large number of muons. Since the number of muons in a muon bundle is not directly measurable it is difficult to associate the total measured energy in-ice to the true energy of a single high-energy muon. In order to ensure a good energy reconstruction, we define signal and background as the following:

$$
\begin{array}{r}
\text { signal } \equiv \text { single muon event }:=\frac{E_{\mu, \max }}{E_{\mu, \text { bundle }}}>0.5, \\
\text { background } \equiv \text { muon bundle event }:=\frac{E_{\mu, \max }}{E_{\mu, \text { bundle }}} \leq 0.5,
\end{array}
$$

with the energy $E_{\mu, \max }$ of the most energetic muon in the muon bundle and $E_{\mu \text {,bundle }}$ as the total energy of the muon bundle. Muons with an energy $\gtrsim 30 \mathrm{TeV}$ at the surface will almost always be 
the leading muon in the shower (compare [13]). The correlation and ratio of reconstructed and true energy of the most energetic muon in the shower for single muon events after the minimum quality cuts are shown for the standard simulations in Figure 1.
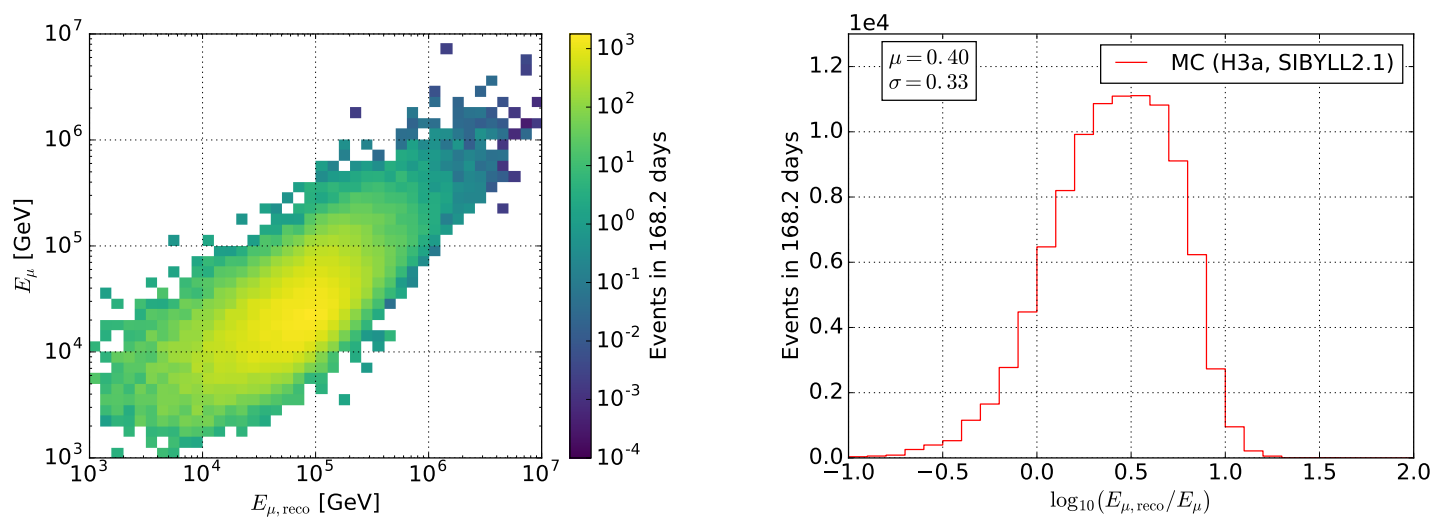

Figure 1: Correlation (left) and ratio (right) of reconstructed and true energy of the most energetic muon in the shower for single muon events after the minimum quality cuts using standard simulations.

The Pearson product-moment correlation coefficient between true and reconstructed muon energy is $r_{\text {Pearson }}=0.75$ with the mean of the ratio on a logarithmic scale $\mu=0.40$ and the standard deviation of the distribution $\sigma=0.33$. In order to obtain a sample of single muon events, the standard simulations are used to train a random forest classifier that separates between single muon events and muon bundle events. Before training the model, two additional straight cuts on the ratio $q_{\mathrm{max}} / Q_{\mathrm{tot}}$ between the maximum charge $q_{\max }$ deposited in a single digital optical module (DOM) divided by the total charge $Q_{\text {tot }}$ deposited in IceCube and on the direct length $L_{\mathrm{dir}}$ of the track given by DOMs that are hit within a certain time window around the first hit of an event are applied. The first cut, $q_{\max } / Q_{\text {tot }}<0.4$, removes events where the total brightness in the detector is dominated by a single DOM and which are not appropriately described by simulations. The second cut, $L_{\mathrm{dir}}>440 \mathrm{~m}$, ensures a decent track reconstruction. For the random forest classification, the implementation from scikit-learn [14] is used. The random forest is trained using 16 attributes, 200 estimators and 4 features per node. The resulting separation power can be seen in Figure 2 for the standard simulations after the minimum quality cuts and the two additional cuts described in this section. For a score $\gtrsim 0.5$ the sample is dominated by single muon events. In order to ensure the robustness of the model, a 5-fold cross-validation yielding values for purity and efficiency depending on the chosen cut on the random forest score is implemented; the resulting values can be seen in Figure 3.

\section{Differential Energy Spectrum}

\subsection{Unfolding}

Whereas for the differential energy spectrum of atmospheric muons their energy at production in the atmosphere is of interest, the muon energy is actually measured in-ice. In order to account for effects like a limited energy resolution and stochastic energy losses during the propagation 


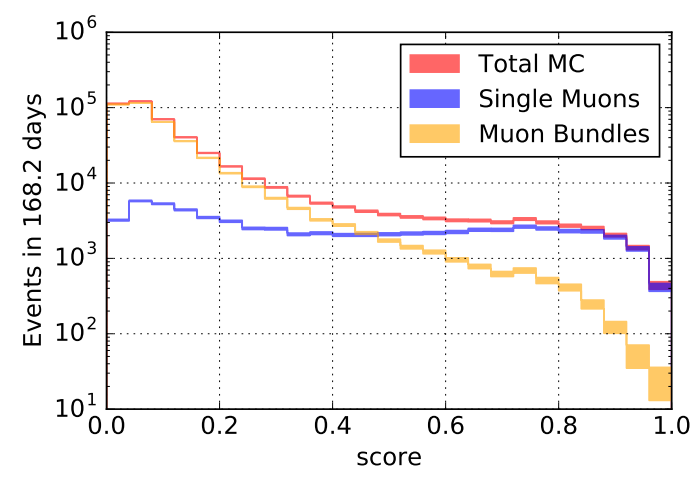

Figure 2: Distribution of the classification score for the standard simulations after the minimum quality cuts and the two additional cuts described in this section.

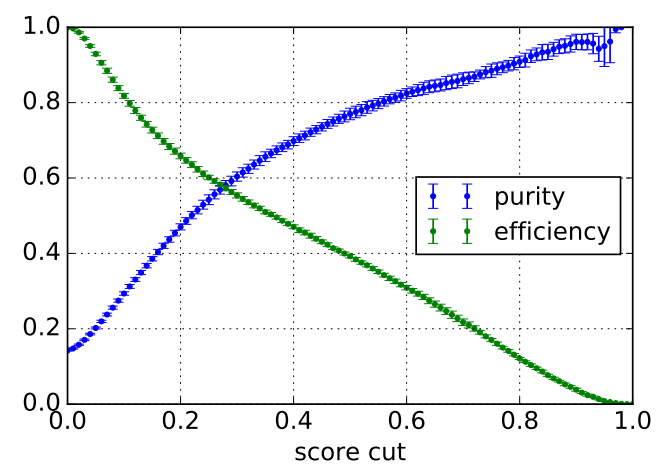

Figure 3: Result of the 5-fold cross-validation of the random forest model showing purity and efficiency as functions of the chosen cut on the random forest score.

of the muons through the ice, the resulting spectrum of the observed muon energy is unfolded using the software package TRUEE [15]. The unfolding is applied to the data after applying a cut (score $>0.55$ ) on the output score of the random forest from Section 3.2, resulting in a purity of $(79.7 \pm 1.3) \%$ and an efficiency of $(35.1 \pm 0.5) \%$. The unfolding is performed in 9 logarithmic energy bins with five bins per decade in the energy range $3.8<\log _{10} E_{\mu} / \mathrm{GeV}<5.6$ and uses the three observables $E_{\mu, \text { reco }}, L_{\mathrm{dir}}$ and $\theta_{\text {zen }}$. The chosen parameters for the unfolding are 4 degrees of freedom and 9 knots. These parameters determine the strength of the regularization (see [15] for a detailed discussion) and were found to work well. In order to estimate the uncertainty of the result due to limited statistics in the simulations, the unfolding is repeated ten times using resampled simulation datasets, yielding an uncertainty $\sigma_{\mathrm{MC}}$, which is then combined with the statistical error $\sigma_{\text {stat }}$ to obtain the overall uncertainty in each bin:

$$
\sigma_{\text {stat } / \mathrm{MC}}=\sqrt{\sigma_{\text {stat }}^{2}+\sigma_{\mathrm{MC}}^{2}}
$$

\subsection{Acceptance Correction}

Only events that trigger IceCube and pass all selection steps are represented in the final sample and thus represented in the unfolded spectrum. In order to correct for the limited acceptance due to these effects, an external simulation dataset [16] is used for obtaining the surface flux predicted for Sibyll 2.1 in the zenith range that is covered by the sample after applying all cuts ( $\left.\cos \theta_{\text {zen }}>0.88\right)$.

\subsection{Spectrum}

The unfolded datapoints with errorbars $\sigma_{\text {stat/MC }}$ compared to different predictions and a previous all-sky result can be seen below. Figure 4 compares the datapoints to the simulated conventional muon flux at the surface from [16], a semi-analytical prediction for the prompt contribution to the muon flux based on the model by Enberg, Reno and Sarcevic [17] using the reweighting approach discussed in [13] as well as a best-fit linear combination of these two contributions. Figure 5, on the other hand, compares the unfolded datapoints $\left(\cos \theta_{\text {zen }}>0.88\right)$ to the best-fit power law from [13] describing the average all-sky flux above approximately $15 \mathrm{TeV}$. The difference in the 


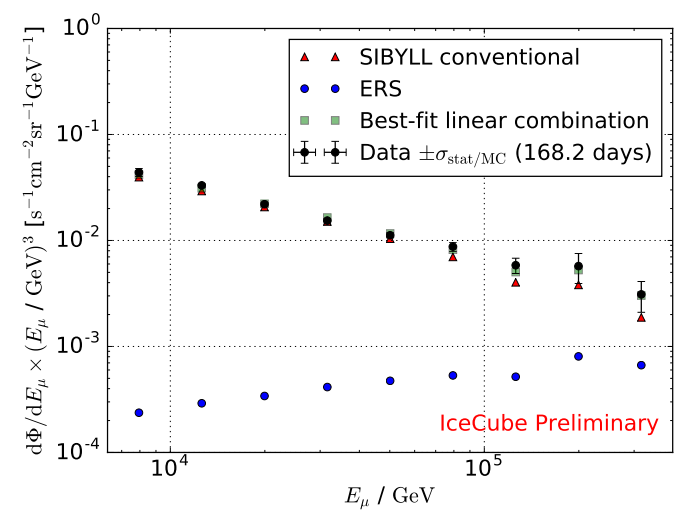

Figure 4: Plot showing the unfolded datapoints (black points), a prediction for the conventional flux from [16] (red triangles), for the prompt flux based on the model from [17], using the reweighting described in [13] (blue points), and the best-fit linear combination (green squares). All datapoints describe the flux in the zenith range $\cos \theta_{\text {zen }}>0.88$.

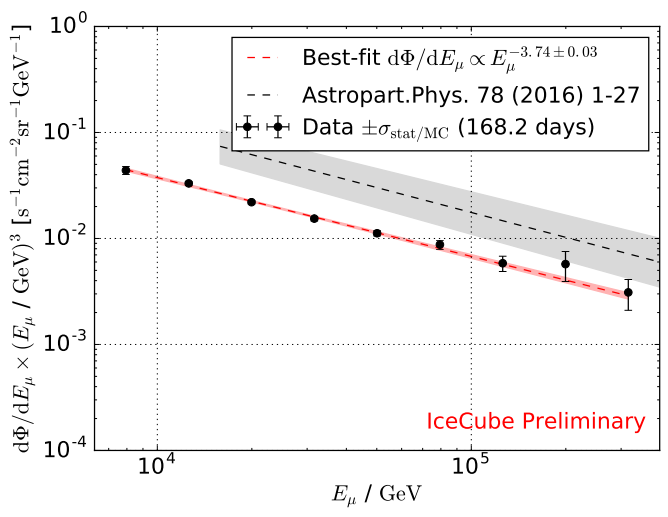

Figure 5: Plot showing the same unfolded datapoints as in Figure 4 (black points), a best-fit power law describing the data (dashed red line) and, for comparison, the best-fit power law from [13] for the average all-sky flux (dashed black line). Shaded areas indicate the uncertainty of the fit parameters (for [13] this includes systematic uncertainties).

The unfolded data points are also fitted to a power law with the best-fit in the zenith range $\cos \theta_{\text {zen }}>$ 0.88 and the energy range $3.8<\log _{10} E_{\mu} / \mathrm{GeV}<5.6$ as the following:

$$
\frac{\mathrm{d} \Phi}{\mathrm{d} E_{\mu}}=9.0_{-0.3}^{+0.3} \times 10^{-17} \mathrm{~s}^{-1} \mathrm{~cm}^{-2} \mathrm{sr}^{-1} \mathrm{GeV}^{-1} \times\left(\frac{E_{\mu}}{50 \mathrm{TeV}}\right)^{-3.74 \pm 0.03},
$$

with $\chi^{2} /$ ndof $=3.6 / 7$. In order to compare the datapoints against the conventional and prompt predictions in Figure 4, a superposition of the form

$$
f\left(E_{\mu}\right)=a_{\mathrm{conv}} \times\left(\frac{\mathrm{d} \Phi}{\mathrm{d} E_{\mu}}\right)_{\text {conv }}+a_{\mathrm{prompt}} \times\left(\frac{\mathrm{d} \Phi}{\mathrm{d} E_{\mu}}\right)_{\text {prompt }}
$$

is fitted to the data. The best-fit result yields $a_{\text {conv }}=1.05 \pm 0.03$ and $a_{\text {prompt }}=1.58 \pm 0.91$ with $\chi^{2} /$ ndof $=6.0 / 7$.

\section{Effective Feynman-x}

\subsection{Phase Space}

In Figure 6, the phase space in true Monte-Carlo variables with respect to primary cosmic ray energy and effective Feynman- $x$ and after the minimum quality cuts is shown for single muon events (left) and muon bundle events (right).

Single muons, carrying most of the energy in the bundle, are also found at larger values of the effective Feynman- $x\left(x_{\mathrm{F}, \mathrm{aff}}^{\mathrm{lab}} \gtrsim 10^{-3}\right)$ and small primary energies $\left(E_{\mathrm{cr}} \lesssim 10^{8} \mathrm{GeV}\right)$. Muon bundle events, on the other hand, are shifted towards higher primary energies and smaller effective Feynman- $x$. 

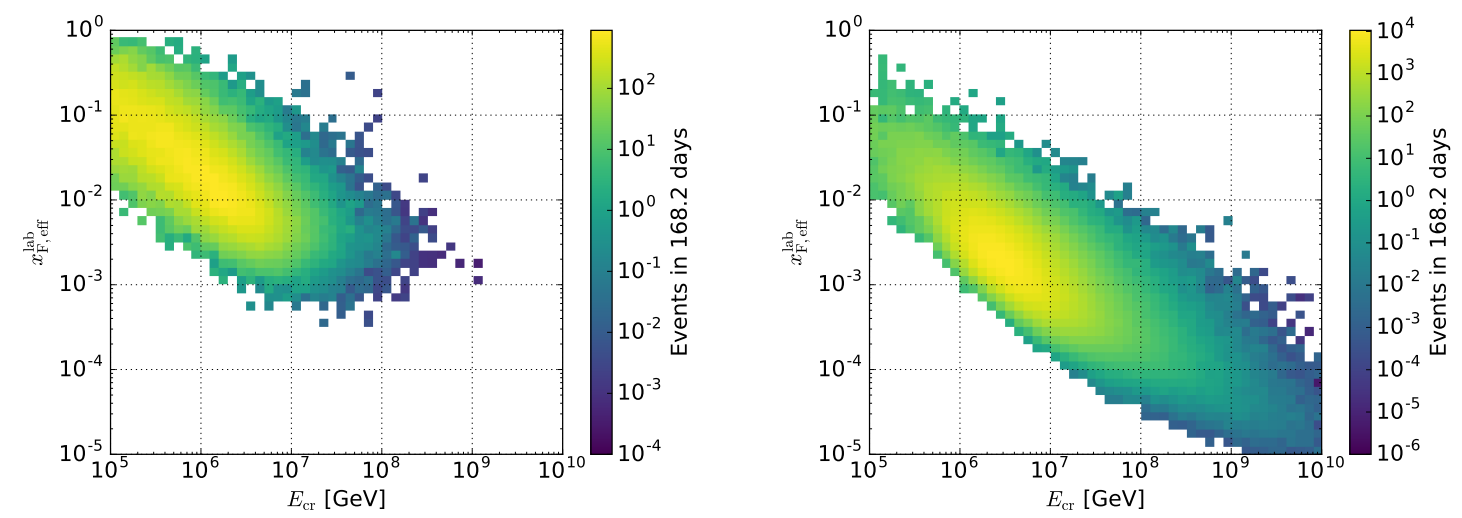

Figure 6: Phase space in true MC variables primary energy and effective Feynman- $x$ for single muon events (left) and muon bundle events (right) for the standard simulations after the minimum quality cuts.

\subsection{Reconstruction}

The effective Feynman- $x$ of atmospheric muons is reconstructed using a machine-learning based regression. The regression is trained using the dedicated simulations described in Section 2 after applying a cut (score $>0.8$ ) on the output score of the random forest from Section 3.2, resulting in a purity of $(90.8 \pm 1.5) \%$ and an efficiency of $(12.1 \pm 0.3) \%$. Again, the implementation from scikit-learn [14] is used, employing 23 overall features, 400 estimators and 6 features per node. The resulting correlation and ratio between reconstructed and true effective Feynman- $x$ can be seen in Figure 7. The Pearson product-moment correlation coefficient of true and reconstructed effective Feynman- $x$ is $r_{\text {Pearson }}=0.62$ with the mean of the ratio on a logarithmic scale $\mu=0.11$ and the standard deviation of the distribution $\sigma=0.27$.
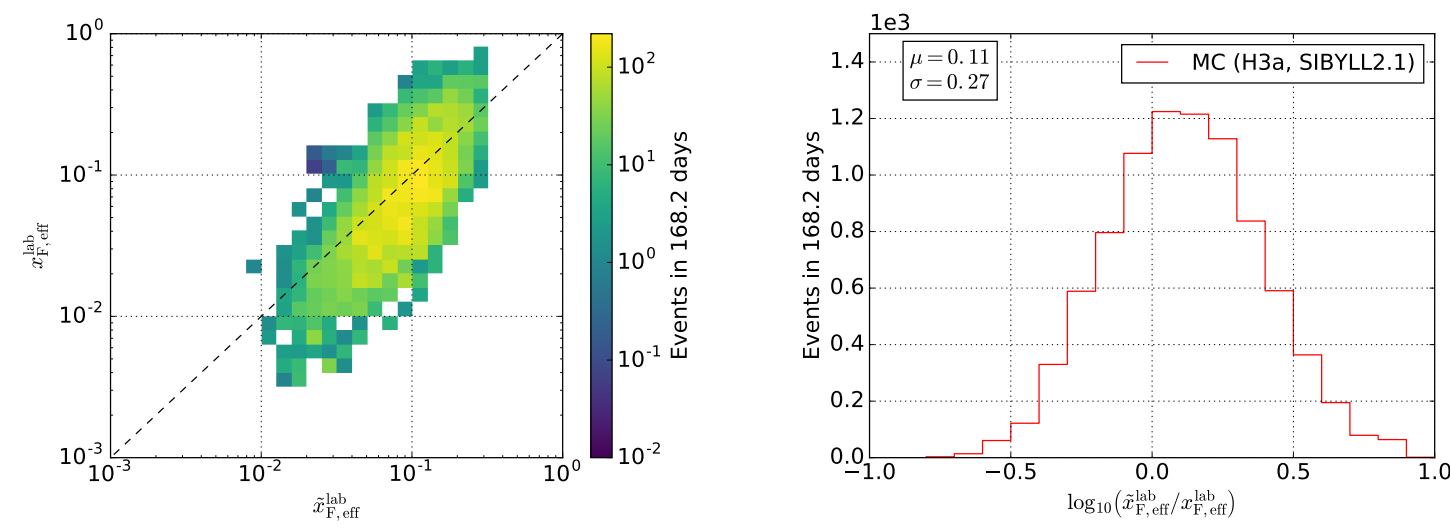

Figure 7: Correlation (left) and ratio (right) of reconstructed and true effective Feynman- $x$ after the minimum quality cuts, the additional cuts described in Section 3.2 and a cut on the random forest classification score (score $>0.8$ ) using the dedicated simulations described in Section 3. The dashed black line in the left plot indicates a line through the origin. 


\section{Conclusion and Outlook}

High-energy atmospheric muons were studied with respect to their differential energy spectrum and the fraction of energy they take from the primary cosmic ray. The differential energy spectrum in the zenith range $\cos \theta_{\text {zen }}>0.88$ and the energy range $3.8<\log _{10} E_{\mu} / \mathrm{GeV}<5.6$, using 168.2 days of detector livetime, was found to follow a power law with $\mathrm{d} \Phi / \mathrm{d} E_{\mu} \propto E_{\mu}{ }^{-3.74 \pm 0.03}$, which is in agreement with the all-sky result from [13]. A linear combination of conventional (Sibyll 2.1) and prompt (ERS) predictions was fitted to the unfolded data points, yielding $a_{\text {conv }}=$ $1.05 \pm 0.03$ and $a_{\text {prompt }}=1.58 \pm 0.91$ as best-fit multiples of these predictions. The stated uncertainties are statistical only and do not contain systematic effects. A multivariate method was presented, capable of reconstructing the fraction of the primary cosmic ray energy that is transferred to the most energetic muon in the shower with a correlation coefficient of $r_{\text {Pearson }}=0.62$ and the standard deviation of the ratio of true and reconstructed values $\sigma=0.27$. In the future, these studies will be extended by using more data, by increasing the effective livetime of the simulations and by studying systematic uncertainties on the hadronic interaction models, the mass composition of cosmic rays, ice-properties and the snow effect on IceTop.

\section{References}

[1] IceCube Collaboration, M. G. Aartsen et al., J. Instrum. 12 (2017) P03012.

[2] IceCube Collaboration, M. G. Aartsen et al., Science 342 (2013) 1242856.

[3] J. I. Illana et al., Astropart. Phys. 34 (2011) 663-673.

[4] A. Fedynitch et al., Eur. Phys. J. 99 (2015) 08001.

[5] T. K. Gaisser, Astropart. Phys. 35 (2012) 801-806.

[6] D. Heck et al., Tech. Rep. FZKA 6019 (1998).

[7] E.-J. Ahn et al., Phys. Rev. D80 (2009) 094003.

[8] T. T. Böhlen et al., Nucl. Data Sheets 120 (2014) 211-214.

[9] A. Ferrari et al., CERN 2005-10 (2005), INFN/TC 05/11, SLAC-R-773 (2005).

[10] L. Breiman, Mach. Learn. 45 (2001) 5-32.

[11] J. Ahrens and et. al., Nucl. Instr. Meth. Phys. Res. A524 (2004) 169-194.

[12] IceCube Collaboration, R. Abbasi et al., Nucl. Instr. Meth. Phys. Res. A703 (2013) 190198.

[13] IceCube Collaboration, M. G. Aartsen et al., Astropart. Phys. 78 (2016) 1-27.

[14] F. Pedregosa et al., J. Mach. Learn. Res. 12 (2011) 2825-2830.

[15] N. Milke et al., Nucl. Instr. Meth. Phys. Res. A697 (2013) 133-147.

[16] A. Fedynitch, J. Becker Tjus, and P. Desiati, Phys. Rev. D86 (2012) 114024.

[17] R. Enberg, M. H. Reno, and I. Sarcevic, Phys. Rev. D78 (2008) 043005. 